\title{
Traditional Chinese Medicine Based On Tongjiang Methodology Combined With Proton Pump Inhibitors (PPIs) De-Escalation In Treating Non-Erosive Reflux Disease: A Study Protocol For A Multicentred, Randomized Controlled Clinical Trial
}

\section{Xia Li (D418336740@qq.com )}

Traditional Chinese Medicine Gastroenterology Department of China-Japan Friendship Hospital, Beijing 100029, China https://orcid.org/0000-0002-7121-7958

\section{Haomeng Wu}

The Second Clinical College of Guangzhou University of Chinese Medicine, Guangzhou 510120, China

\section{Ting Chen}

Xiyuan Hospital Affiliated to China Academy of Traditional Chinese Sciences, Beijing Institute of Spleen and Stomach Disease of Traditional Chinese Medicine, Beijing 100091, China

\section{Beihua Zhang}

Xiyuan Hospital Affiliated to China Academy of Traditional Chinese Sciences, Beijing Institute of Spleen and Stomach Disease of Traditional Chinese Medicine, Beijing 100091, China

\section{Xiaoshuang Shi}

Institute of Acupuncture and Moxibustion, Center for Post-doctoral Studies, China Academy of Chinese Medical Sciences, Beijing 100700, China

\section{Jinxin Ma}

Department of Gastroenterology, Peking University Traditional Chinese Medicine Clinical Medical School (Xiyuan), Beijing 100091, China

\section{Jiaqi Zhang}

Xiyuan Hospital Affiliated to China Academy of Traditional Chinese Sciences, Beijing Institute of Spleen and Stomach Disease of Traditional Chinese Medicine, Beijing 100091, China

\section{Xudong Tang}

China Academy of Traditional Chinese Sciences, Beijing Institute of Spleen and Stomach Disease of Traditional Chinese Medicine, Beijing 100091, China

\section{Fengyun Wang}

Xiyuan Hospital Affiliated to China Academy of Traditional Chinese Sciences, Beijing Institute of Spleen and Stomach Disease of Traditional Chinese Medicine, Beijing 100091, China

\section{Study protocol}

Keywords: nonerosive reflux disease (NERD), TCM granules, PPI, Randomized controlled trial (RCT), protocol 
Posted Date: September 14th, 2021

DOI: https://doi.org/10.21203/rs.3.rs-826755/v1

License: (c) (i) This work is licensed under a Creative Commons Attribution 4.0 International License. Read Full License 


\section{Abstract}

Background: Non-erosive reflux disease (NERD) is characterized by typical gastroesophageal reflux symptoms, such as heartburn and regurgitation, but an absence of oesophageal mucosal damage during upper gastrointestinal endoscopy. Although proton pump inhibitors (PPIs) are the first line therapy, almost $50 \%$ of patients with NERD fail to respond to this treatment. Traditional Chinese medicine (TCM) can better relieve the symptoms of NERD. Therefore, a randomized controlled trial (RCT) was designed to investigate the efficiency of TCM granules based on Tongjiang (TJ) methodology combined with PPIs de-escalation therapy for NERD patients who did not respond to PPIs alone.

Method: This multicentred, double-blinded, RCT with two parallel groups will recruit 174 participants who will be randomized into the TCM granules combined with PPIs de-escalation group $(n=87)$ and the TCM granules placebo combined with PPIs de-escalation group $(n=87)$. Both groups of participants will receive 6 weeks of treatment and 4 weeks of follow-up, and all participants will be assessed for related symptoms, mental health status and quality of life at each visit. The primary outcome measurements include Visual Analogue Scale (VAS) for heartburn and regurgitation and the major symptoms scale. The secondary outcome measurements include PPI withdrawal rate, symptom recurrence rate, minor symptoms scale, SF-36, PRO, SAS, SDS, GERD-HRQL and TCM syndromes scales.

Discussion: Previous research has shown that TCM is capable to alleviate NERD symptoms. This trial will help to provide a better understanding of the synergistic efficiency of the combination of TCM and PPIs, to explore whether the dosage of PPIs can be reduced after the supplement of TCM granules and to provide a feasible plan to reduce dependencies or withdraw NERD patients from PPIs. The outcome of this trial is expected to reduce the symptom recurrence rates, lessen patients' physical and psychological burdens, and achieve good social benefits.

Trial registration number: Clinicaltrials.gov: NCT04340297

\section{Background}

Gastroesophageal reflux disease (GERD) is one of the most common chronic progressive upper gastrointestinal tract disorders of the oesophagus, characterized by heartburn and regurgitation symptoms [1]. The prevalence of this disease ranges from $2.5 \%$ to more than $25 \%$ according to individual cross-sectional surveys [2]. GERD can be divided into several phenotypes: erosive esophagitis (EE), Non-erosive reflux disease (NERD), and Barrett's oesophagus (BE) $[3,4]$. NERD is the most frequent phenotype of gastroesophageal reflux disease (GERD). Rome IV consensus defines NERD as "the presence of abnormal acid exposure time (AET) with or without refluxsymptom association on ambulatory reflux monitoring performed off anti-secretory therapy" [5]. It accounts for approximately $70 \%$ of GERD patients and is characterized by typical symptoms and the lack of any visible endoscopic findings of oesophageal mucosal lesions [6,7]. The majority of patients with NERD have weakly acidic reflux [8]. Some studies have shown that the function of the oesophageal mucosal barrier is not significantly altered in NERD patients [9]. NERD is a refractory gastrointestinal disease, and patients often need long-term treatment to control their symptoms. Proton pump inhibitors (PPIs), a type of acid-suppressive drug, are considered the first-line treatment drugs for NERD. There are obvious deficiencies in treatment guideline and safety of PPIs, which demonstrated the following problems in clinical practice: approximately $50 \%$ of patients 
with weakly acidic reflux respond partially or not at all to PPIs [10]; $20-30 \%$ of patients treated with a standard PPI dose for troublesome GERD symptoms experience persistent heartburn and/or regurgitation $[11,12]$ and more than 75\% NERD patients may experience symptomatic relapse within six months of stopping treatment so they receive long-term treatment to control symptoms $[13,14]$; long-term use of PPIs can cause many adverse events, such as indigestion, fundic gland polyps, atrophic gastritis, and gut microbiota imbalance $[15,16]$, as well as PPIs resistance or dependence. This life-long dependency leads to a huge psychological and physical burden for the patient [17].

Due to insufficient patient-response-to treatments and various undesirable negative side effects, more and more scientific researchers in the medical area have begun to consider PPIs de-escalation therapy in recent years. PPIs de-escalation therapy means gradually reducing dosage and patients' dependence on PPIs according to the outcome and symptoms changes of patients. Multiple strategies may be used to de-escalate (i.e. stop or reduce) PPIs, including abrupt PPI withdrawal, step down therapy, and $\mathrm{H}_{2}$ blocker (i.e. famotidine) substitution. However, one Randomized controlled trial (RCT) study showed that about $68 \%$ patients experienced symptom recurrence after discontinuation of long-term PPIs therapy [18], thus, meaning that stopping PPIs is not sufficient to control patients' symptoms in a long term period.

The use of complementary and alternative approaches has proven to have an effect on the treatment of NERD $[19,20]$. In recent years, numerous studies have evaluated the clinical effectiveness of traditional Chinese Medicine (TCM) herbals for the treatment of NERD. A review article showed that in addition to their acidsuppressive properties, anti-inflammatory and antioxidant activity of the herbal remedies, these herbal medicine seem to provide a safer and more effective treatment compared to their pharmaceutical drug counterparts. Herbal remedies used in human studies have led to the alleviation of heartburn and regurgitation related to NERD where PPIs treatment has failed to show a promising effect. Moreover, herbal remedies appeared to have a longer-lasting therapeutic effect than conventional anti-secretory agents have [19]. A systematic review has reported that the total effective rate of TCM alone was superior to single PPIs or prokinetics for their clinical efficacy. TCM also has advantages in reducing recurrence rate and adverse events like constipation, faint headache experience and nausea [21]. Although TCM has been examined for its ability to alleviate PPI-refractory symptoms, including the reduction of recurrence of these symptoms as well as improve the quality of life of patients; a high-level evidence-based support and thorough understanding of the underlying mechanisms of the action of TCM herbal remedies were lacked [22].

Tongjiang (TJ) methodology is used in TCM to treat functional gastrointestinal diseases. TJ literally means unobstructed and descent, and the main goals of TJ methodology in TCM are to regulate visceral dysfunction, adjust the imbalance between internal and external energies, restore the physiological functions of the viscera, revitalise the mind, spirit and body for a holistic well-being balance. Herbal granules based on TJ methodology can help regulate cell-to-cell tonic equilibrium and improve the overall Qi and food passing unimpeded through the entire gastrointestinal tract. Prior to this trail, we have performed an RCT study on the efficacy of TJ granule to improve the quality of the results and to provide estimates for further sample size calculation. Results showed that TJ granule could improve the symptoms which included heartburn, chest pain, acid or bitter tastes in mouth, and nausea. On the quality of life, it could also improve the domains of physical functioning and general health, and no adverse event was found [23]. 
For most common patients presented with NERD [17], we will use TCM granules based on the TJ methodology in cooperation with PPIs de-escalation therapy to treat NERD. We designed this rigorous multicentre, doubleblinded, RCT using TJ methodology granules with PPIs de-escalation therapy, to explore its therapeutic efficiency, measure recurrence rate, understand the mechanisms of the therapy and estimate PPI withdrawal rate.

Unlike the use of PPIs alone, we aimed to independently examine the efficiency and safety of TJ methodology granules combined along with PPIs de-escalation therapy for NERD patients with regard to NERD-related symptoms and symptom distress including regurgitation and heartburn, health-related quality of life, mental health, and measurable biomarkers. We will analyze the PPIs withdrawal rate, measure symptom recurrence rate and predict whether the dosage of PPIs can be significantly reduced after the supplement of TJ methodology granules for NERD patients who had been taking PPIs for a long period of time or whose previous curative effect is not satisfactory. We will also explore some of the common mechanisms of this therapy for NERD based on observations of the changes in patients' gut microbiota.

\section{Method}

\section{Study design}

This trial will adopt a multicentre, double-blinded, randomized controlled trial with two parallel groups, to explore the efficiency of the TJ methodology granules combined with PPIs de-escalation for NERD. The protocol will be described in accordance with Recommendations for Interventional Trials 2013 guidelines [24]. The details of the study design are shown in Figure 1.

\section{Study setting}

This multicentre, double-blind, RCT will be conducted at six hospitals in China (Xiyuan Hospital of China Academy of Chinese Medical Sciences; Guangdong Provincial Hospital of Traditional Chinese Medicine; Wuhan No.1 Hospital; The First Affiliated Hospital of Tianjin University of Traditional Chinese Medicine; Traditional Chinese Medicine Hospital of Kunshan; Liuzhou Hospital of Traditional Chinese Medicine). A total of 174 participants will be recruited from the hospital outpatient departments, hospital websites and posters. Participants will be divided into three groups according to their different TCM syndromes, and those with the same syndrome will be divided into the intervention group and the control group at a 1:1 ratio.

\section{Eligibility criteria}

All participating patients must first be judged by TCM physicians to determine whether they meet the following inclusion and exclusion criteria.

\section{Inclusion criteria}

(1) The diagnostic criteria of NERD according to the China Consensus Opinion on Gastro-oesophageal Reflux Disease, 2014 edition and treatment with PPIs for more than 8 weeks and whose curative effect is not satisfactory; (2) The diagnostic criteria of stagnation of liver and stomach heat syndrome, spleen deficiency and dampness-heat syndrome and intermingled heat and cold syndrome in TCM; (3) Agreement to participate in the clinical trial and sign the informed consent form; (4) Age from 18 to 65 years old. 


\section{Exclusion criteria}

(1) Pregnant or breastfeeding females or those who are planning pregnancy; (2) Other chronic GI diseases like $\mathrm{RE}$ or BE or primary diseases such as peptic ulcers, achalasia, pyloric obstructions, and oesophageal cancer that can cause gastroesophageal reflux; (3) Severe cardio-cerebrovascular system diseases, liver, kidney, hematopoietic system diseases, psychiatric diseases and tumours; (4) Anti-reflux surgery (fundoplication or endoscopic minimally invasive surgery) and other upper gastrointestinal surgery records; (5) History of allergies or drug and food allergies appearing during the trials; (6) Inability to describe self-symptoms or to cooperate with treatment; (7) Abuse of alcohol or drugs, frequent changes in the working environment and tendency to dropout; (8) Participation in other clinical trials within 4 weeks.

\section{Dropout criteria}

(1) Decision to exit the trial himself/herself; (2) Loss to follow-up; (3) Poor compliance; (4) Diagnosis with other TCM syndromes or an order to withdraw by the TCM physicians.

For the occurrence of patients presented with the above situation, we will consider making a suspension. In cases of dropout, the reason should be explained. If there are baseline pharmacodynamic data, the last main therapeutic effect index can be transferred to the final results for statistical analysis, and the CRF should be retained.

\section{Intervention}

Each centre's treatment of participants will be provided by licensed TCM physicians. TCM physicians will distribute granules to participants tailored according to their symptoms under the guidance of the TJ methodology. To reduce intervention bias, TCM physicians have conducted relevant training. Our record physicians must study and be familiar with the scales and questionnaires in this trial, and learn the skills to guide participants to complete them appropriately. The granule-distributing physicians arrange and distribute the granules accordingly. In addition to that, we have a chief physician responsible for overseeing the entire process.

\section{Intervention group}

Based on the curative effect of treating NERD in clinical practice, we selected three kinds of TCM granules based on TJ methodology. According to the different TCM syndromes, participates with stagnation of liver and stomach-heat syndrome will be treated with Tongjiang (TJ) granule, those with spleen deficiency and dampnessheat syndrome will be treated with Jianpiqinghua (JQ) granule and those with intermingled heat and cold syndrome will be treated with Wenpiqingwei (WQ) granule. TCM granules are concentrated herbal particles obtained by the mean of spray drying the hot water extract of a mixture of raw herbs. TCM granules are extracted with water to make an aqueous extract or decoction used for treatment. Each bag of granules (approximately 20g) will be dissolved in $200 \mathrm{ml}$ water and taken three times a day, 1 hour after meal. Participants in all three groups cooperated with PPIs de-escalation therapy. From week 1 to week 2 of treatment, the dose of PPIs will be half of the original dose, once a day, taken before bedtime. The PPIs will be replaced with famotidine $20 \mathrm{mg}$ from week 3 to week 4 , taken before bedtime. From week 5 to week 6 , acid-suppressive drugs will be withdrawn, and only TCM granules will be administered. There will be 6 weeks of treatment and 4 weeks of follow-up. 


\section{Control group}

Patients will be treated with the corresponding TJ methodology granules placebo. The placebo granules consist of cyclodextrin and $5 \%$ real TJ theory granules to imitate the color and smell of the real counterpart. Both herbal granules and placebo are manufactured by China resources sanjiu medical and pharmaceutical co., Itd. (Hefei, China; Batch number: 2004902) according to the standards of the Good Manufacturing Practice. The physical properties of the placebo, such as appearance, size, colour, dose form, weight, taste, and smell, will be similar to those of the TCM granules. The PPIs de-escalation therapy will be the same as that for the intervention group.

\section{Cointerventions}

One week prior to the start of treatment, patients will be required to stop their intake of other drugs related to TCM therapies of NERD, however, acid-suppressive drugs intake will remain unchanged. During the study period, patients will be forbidden to add any drugs other than study therapies without permission. During the 1-4 weeks of the treatment period, if the participant's symptoms increase after the reduction of PPIs according to the study protocol and cannot be tolerated, participant can temporarily use almagate suspension or almagate chewable tablets and record the combined medication. During the treatment period of 5-6 weeks, if the participant is unable to tolerate the symptoms after stopping the acid suppressive drugs, participant will be terminated from the trial and recorded, and will be treated with their original treatment therapy.

\section{Timeline}

The entire trial period will last 10 weeks (the treatment period is 6 weeks and the follow-up period will last 4 weeks). Participants will need to stop using drugs related to the treatment of NERD during pre-screening and screening, complete a physical safety examination and sign an informed consent form. At baseline, participants will need to complete the first assessment, and then randomly divided into an intervention group and a control group. During the treatment phase, TCM physicians will instruct the participants on how to take the therapy, and then evaluate compliance and outcome indicators after 2, 4 and 6 weeks of treatment. The follow-up period will last 4 weeks after the end of treatment. Details are shown in Table 1.

\section{Sample size estimation}

According to the preliminary trial results from the team and to literature reports, the average effective rate of traditional Chinese medicine treatment for NERD is $90 \%$ [25]. The literature reports that the effective rate of PPIs in the treatment of NERD is between $50 \%$ and $90 \%[4,26]$, so we used an average of $70 \%$. The trial intends to conduct a superiority test of two independent sample proportions, using a margin of 0.02 , an alpha risk of 0.05 and a beta risk of 0.20 . Assuming an attrition rate of approximately $20 \%$. The estimated sample size is $\mathrm{N}=174$, including 87 cases in the intervention group and 87 cases in the placebo group.

\section{Randomization and blinding}

In this trial, centre stratification will be used; each centre will use the block randomization method, and qualified participants will be divided into intervention and control groups in a 1:1 ratio for grouping. The stratified randomization will be completed by a statistician through the PROC PLAN procedure in Strategic Applications Software (SAS) V.9.2, and the results of the random allocation will be published through the network central 
random allocation system. Groups will be placed into sealed brown envelopes, and the clinical research coordinator will number the envelopes sequentially and maintain them.

The patient's sequence number and corresponding grouping will comprise the first-level blind code and, the medicine code will comprise the second-level blind code; each patient's medicine code will be randomly assigned. All operations will be completed according to the established SOP guidelines. All codes will be kept in the Good Clinical Practice (GCP) Center of Xiyuan Hospital. The TCM practitioners and statisticians administering the treatment will be blinded to the grouping.

In the case of serious adverse events or complications in the clinical trials, blinding should be unsealed urgently, and cases of unblinding should be treated as dropout participates.

Table 1 Timeline of the trial. 
study period

\begin{tabular}{|c|c|c|c|c|c|c|c|c|c|c|}
\hline \multirow[b]{2}{*}{ outcomes } & \multirow{2}{*}{$\begin{array}{l}\text { screen- } \\
\text { ing } \\
\text { week } \\
-1\end{array}$} & \multirow{2}{*}{$\begin{array}{l}\text { base- } \\
\text { line } \\
\text { week } \\
0\end{array}$} & \multicolumn{6}{|c|}{ treatment } & \multicolumn{2}{|c|}{ follow-up } \\
\hline & & & $\begin{array}{l}\text { week } \\
1\end{array}$ & $\begin{array}{l}\text { week } \\
2\end{array}$ & $\begin{array}{l}\text { week } \\
3\end{array}$ & $\begin{array}{l}\text { week } \\
4\end{array}$ & $\begin{array}{l}\text { week } \\
5\end{array}$ & $\begin{array}{l}\text { week } \\
6\end{array}$ & $\begin{array}{l}\text { week } \\
8\end{array}$ & $\begin{array}{l}\text { week } \\
10\end{array}$ \\
\hline \multicolumn{11}{|l|}{$\begin{array}{l}\text { Screening and } \\
\text { enrolment }\end{array}$} \\
\hline $\begin{array}{l}\text { Informed consent } \\
\text { form }\end{array}$ & 0 & & & & & & & & & \\
\hline $\begin{array}{l}\text { Eligibility for } \\
\text { study }\end{array}$ & ○ & & & & & & & & & \\
\hline $\begin{array}{l}\text { Demographic } \\
\text { and clinical } \\
\text { details }\end{array}$ & 0 & & & & & & & & & \\
\hline $\begin{array}{l}\text { Physical safety } \\
\text { examination }\end{array}$ & 0 & & & & & & & & & \\
\hline Randomization & 0 & & & & & & & & & \\
\hline \multicolumn{11}{|l|}{ Intervention } \\
\hline $\begin{array}{l}\text { TCM } \\
\text { granules/placebo } \\
\text { +PPIs de- } \\
\text { escalation }\end{array}$ & & 0 & & 0 & & 0 & & & & \\
\hline \multicolumn{11}{|l|}{ Assessment } \\
\hline $\begin{array}{l}\text { VAS of heartburn } \\
\text { and reflux }\end{array}$ & & 0 & 0 & 0 & 0 & 0 & 0 & 0 & 0 & 0 \\
\hline $\begin{array}{l}\text { Major symptoms } \\
\text { scale }\end{array}$ & & 0 & 0 & 0 & 0 & 0 & 0 & 0 & 0 & 0 \\
\hline $\begin{array}{l}\text { Minor symptoms } \\
\text { scale }\end{array}$ & & $\bullet$ & - & 0 & 0 & 0 & - & 0 & 0 & 0 \\
\hline $\begin{array}{l}\text { TCM syndromes } \\
\text { scale }\end{array}$ & & ○ & & 0 & & 0 & & 0 & 0 & 0 \\
\hline SF-36 & & 0 & & 0 & & 0 & & 0 & ○ & 0 \\
\hline $\begin{array}{l}\text { PRO for chronic } \\
\text { gastrointestinal } \\
\text { diseases }\end{array}$ & & 0 & & 0 & & 0 & & $\bullet$ & 0 & 0 \\
\hline SAS & & 0 & & 0 & & 0 & & 0 & 0 & 0 \\
\hline SDS & & 0 & & ○ & & 0 & & 0 & 0 & 0 \\
\hline GERD-HRQL & & 0 & & 0 & & 0 & & 0 & 0 & 0 \\
\hline Compliance & & & 0 & 0 & 0 & 0 & 0 & 0 & & \\
\hline Cointerventions & & & - & - & • & • & - & - & & \\
\hline
\end{tabular}


VAS, visual analogue scale; TCM, traditional Chinese medicine; SF-36, the MOS 36-item Short From Health Survey; PRO, Patient-Reported Outcome; SAS, Self-rating Anxiety Scale; SDS, Self-rating Depression Scale; GERD-HRQL, the Gastroesophageal Reflux Disease-Health-Related Quality-Of-Life.

\section{Compliance}

The patient's compliance will be calculated as "actual dose/dose to be taken"; if the compliance is in the range of 0.7 to 1.2, it will be regarded as good compliance. Participants should return unused medicine and empty packaging at each visit, and TCM physicians will count the number of drugs and granules returned and keep a record of lost and unreturned medicines.

\section{Ethics and dissemination}

\section{Patient consent}

The trial is approved by the Ethical Review Committee of Xiyuan Hospital of China Academy of Chinese Medical Sciences. For eligible participants, a TCM physician will provide written informed consent forms. After the physician communicates the details of the study, participants willing to participate in the trial will sign an informed consent form. Participates and the TCM physician will each keep a copy of the form.

\section{Confidentiality}

All materials related to the trial will be safely stored in the restricted access location. All participant case reports and electronic charts will be kept confidential, and only authorized researchers will be able to access the paper version and electronic data set.

\section{Outcomes and analysis}

Participants in both the intervention group and control group will be encouraged to complete the treatment in 6 weeks, and follow-up will continue for 4 weeks. The following outcome indicators will be used to evaluate efficacy and safety. Demographic and clinical details of participants will be collected at the beginning of the trial. Physical safety examinations will be conducted at the beginning and end of the study, including: (1) routine tests of blood, urine and stool, faecal occult blood; (2) liver function tests (ALT, AST) and, kidney function tests (Cr, BUN); and (3) electrocardiograms. Outcome indicators for efficacy evaluation will include the following: (1) a visual analogue scale (VAS) for heartburn and regurgitation; (2) a major symptoms scale; (3) PPIs withdrawal rate and symptoms recurrence rate; (4) a minor symptoms scale; (5) the MOS 36-item Short From Health Survey (SF-36); (6) the Patient-Reported Outcome (PRO) scale for chronic gastrointestinal diseases; (7) Self-Rating Anxiety Scale (SAS); (8) Self-rating depression scale (SDS); (9) the Gastroesophageal Reflux Disease-HealthRelated Quality-Of-Life (GERD-HRQL) scale; (10) the TCM syndromes scale. Scales and questionnaires will be completed at the beginning and end of the trial and at the end of each phase and follow-up. Adverse events will be observed throughout the treatment.

\section{Primary outcomes}

The primary outcomes are the VAS for heartburn and regurgitation and the major symptoms scale. 
(1) The VAS is a line marked with 10 scales, with 0 points to 10 points at both ends. Zero points indicates no symptoms, and 10 points represents the most severe and unbearable symptoms. 0-2 is classified as "basic no symptoms", 3-5 is classified as "mild", 6-8 is classified as "moderate", and 8-10 is classified as "severe" [27-29]. Patients will be given a VAS for regurgitation and heartburn daily through a diary card, and the average score of the two symptoms each week will be evaluated according to the content of the diary card. Participants were defined as responders if for at least $50 \%$ of weeks during the 6 weeks of the trial the weekly VAS score was reduced from baseline by $\geq 50 \%$. We will calculate the percentage of responders. At the same time, the change in VAS score will be compared between groups.

(2) Major symptoms scale: For the main clinical symptoms of NERD, heartburn frequency, heartburn relief time, and regurgitation frequency, the scoring criteria are shown in Table 2.

Table 2 Major symptoms scale.

\begin{tabular}{|c|c|c|c|}
\hline $\begin{array}{r}\text { score } \\
\text { symptom }\end{array}$ & Mild (0) & Moderate (1) & Severe (2) \\
\hline heartburn frequency & 1 day a week & 2-3 days a week & 4-7 days a week \\
\hline heartburn relief time & relieve within $1 \mathrm{~h}$ & $\begin{array}{l}\text { relieve within } \\
1-3 \mathrm{~h}\end{array}$ & $\varangle 3 \mathrm{~h}$ does not relieve even all day \\
\hline regurgitation frequency & 1 day a week & 2-3 days a week & 4-7 days a week \\
\hline
\end{tabular}

\section{Secondary outcomes}

Secondary outcomes are the PPI withdrawal rate and symptoms recurrence rate, scales about minor symptoms, health-related quality of life, mental health and TCM syndromes.

(1) PPIs withdrawal rate and symptoms recurrence rate: During patient follow-up for the 4 weeks after the end of the treatment, according to the above scores, the number of recurrences and corresponding recurrence rates. The PPI withdrawal rate will calculated based on the number of people who no longer use PPIs at follow-up.

(2) Minor symptoms scale: Scoring the frequency and degree of clinical minor symptoms. For the frequency, 0 points indicates no symptoms, 1 points indicates < 1 day per week, 2 points indicates 1 day per week, 3 points indicates 2-3 days per week, 4 points indicates 4-5 days per week, 5points indicates 6-7 days per week. For the degree of symptoms, 1 points means no symptoms, 2 points means symptoms are mild, 3 points means symptoms are moderate, need to take medicine occasionally, 4 points means symptoms are severe, need to take medicine for long time, 5 points means symptoms are very serious, and affecting daily life, need to take medicine for long time. Details are shown in Table 3.

Table 3 Minor symptoms scale. 


\begin{tabular}{l|l} 
symptoms & frequency score degree score \\
\hline non cardiogenic chest pain & \\
\hline epigastric pain & epigastric discomfort \\
\hline belch \\
\hline cough \\
\hline asthma \\
\hline pharyngeal paraesthesia \\
\hline total
\end{tabular}

(3) SF-36: As a concise health questionnaire, the SF-36 comprehensively evaluates the quality of life of the respondents from 8 dimensions including Physical Functioning (PF), Role-Physical (RP), Bodily Pain (BP), General Health (GH), Vitality (VT), Social Functioning (SF), Role-Emotiona (RE), Mental Health (MH). It also contains another health dimension: Health Transition $(\mathrm{HT})$, which is used to evaluate the overall change in health in the past year [30-33].

(4) PRO: This scale is for patients with FGIDs. It contains a total of 35 items, divided into 6 dimensions (regurgitation, dyspepsia, general condition, social function, defecation, psychology) to assess the influencing factors of chronic gastrointestinal diseases. Each item is graded on a 5-point scale, with a maximum of 4 points and a minimum of 0 points. The higher the score, the more severe the symptoms $[34,35]$.

(5) SAS: The SAS scale is a 20-item self-reported assessment device. Each question is scored on a Likert-type scale of 1 to 4 (based on the following replies: "a little of the time," "some of the time," "a good part of the time," and "most of the time"). The total score is obtained by summing the assessment of the 20 items. The total score multiplied by 1.25 gives the standard score. A standard cutoff score of 50 is usually used to diagnose anxiety. The standard score ranges are 25-49 (normal range), 50-59 (mild anxiety), 60-69 (moderate anxiety), and 70 (severe anxiety) [36, 37].

(6) SDS: The Self-rating Depression Scale (SDS) developed by Zung is a norm-referenced measure, used to screen adults for the potential presence of depressive disorders. The scale produces raw scores between 20 and 80 , Zung recommended converting these to Index Scores (which ranged between 25 and 100) by the simple process of multiplying by 1.25 . Zung's recommended cut-off for identifying adults with depressive disorder was index scores of 50 and over [38]. We used in this study that an index score of 53 (raw score 42), this was more appropriate for use with Chinese populations[39].

(7) GERD-HRQL: A total of 16 items are used to evaluate the symptoms of heartburn, regurgitation and the effects of current medication on life. Each item is scored on a 6-point scale, from 0 to 5 points. A score of 0 indicates no symptoms, and a score of 5 indicates that symptoms are incapacitating when performing daily activities $[40,41]$. 
(8) TCM syndromes scale: a 15-item scale based on the Clinical Guideline of New Drugs for Traditional Chinese Medicine. This scale evaluates the changes in TCM symptoms of the digestive tract and the whole body of the participants, including flatulence, stomachache, poor appetite, heartburn, belching, acid reflux, pharyngeal paraesthesia, thirst, distention in the lateral lower abdomen, fatigue, shortness of breath, unwillingness to speak, somatosensory heaviness, fear of cold, and loose stool. Each item is scored, with 1 being asymptomatic and 4 being severe. The higher the overall score, the more severe the overall symptoms [42].

\section{Exploring outcomes}

At baseline and after 6 weeks of treatment, faecal samples will be collected and stored at $-80^{\circ} \mathrm{C}$ for unified testing. The experimenter will extract total DNA from the faecal samples and perform $16 \mathrm{~S}$ rRNA sequencing. Reads will be processed to generate an operational taxonomic unit (OTU) table at $97 \%$ granularity. Through OTU analysis, the detect $\alpha$-diversity, $\beta$-diversity of the faecal microbiome will be detected, and then LEfSe analysis will be used to compare and analyse the data to identify species with significant differences in abundance between groups.

\section{Data management}

We will use both written and electronic CRF. At each time point, participants will complete the CRF under the guidance of a TCM physician. The completed CRF will be entered into the Drug \& Clinical Trial Data Management Platform (developed by the GCP center: http://www.xyedc.com/). The CRF data will be entered in duplicate by two trained TCM physicians. Electronic data will be permanently saved. After the study, the statisticians will be able to download the electronic data for analysis through the platform.

\section{Statistical analysis}

This trial will use intention-to-treat (ITT) analysis as the main method to evaluate the outcome results. To deal with missing outcome variables that remain to be recorded, the last record data will be carried over to the end or a multiple imputation method will be used.

Statistical analysis will be conducted using SPSS 21.0 software. The significance level is established at 0.05 and the limits of the confidence interval are $95 \%$. Descriptive analysis and categorical variables will be described in frequency tables and as percentages or composition ratios; continuous variables will be described in terms of means and standard deviations (SDs), or medians, lower quartiles (P25), upper quartiles (P75), minimum values, and maximum values. Comparative analyse between two groups will be performed for categorical variables using the chi-squared test, Fisher's exact probability method, the Wilcoxon rank-sum test, or the $\mathrm{CMH}$ test. Continuous variables that conform to a normal distribution will be compares using a t-test (for homogeneity of variance between groups, with 0.05 as the test level; if the variance is heterogeneous, the Welch-Satterthwaite ttest will be used), while those that conform to a non-normal distribution will be compared using the Wilcoxon rank-sum test or the Wilcoxon signed-rank test. Hypothesis testing will use the two-sided test uniformly, and $\mathrm{P} \leq$ 0.05 will be considered statistically significant.

\section{Discussion}


To date, there are increasing numbers of patients negatively affected by NERD symptoms, which also impose a heavy economic and life burden to patients [43]. This trial will use TCM granules combined with PPIs deescalation therapy as the intervention group and granule placebo combined with PPIs de-escalation as the control group to study the efficiency and safety of the TJ methodology series granules combined with PPIs deescalation for treating NERD patients. This trial aimed to explore the possibilities whether the dosage of PPIs can be significantly reduced after treatment with TCM granules, and observe the recurrence of the disease after treatment.

Based on multiple clinical researches and scientific systematic reviews in recent years, when compared to the treatment of western medicine alone, TCM has superior advantages in the treatment of functional gastrointestinal diseases, which proven to improve treatment effectiveness rates, reduce recurrence rates and side effects, however, there were just small amount of them analyzing and looked thoroughly into the recurrence rate $[21,22]$. The quality of about $90 \%$ TCM RCTs was considered poor, lacking of descriptions of blinding and randomization protocols in studies, neglecting stringent diagnostic guidelines and therapeutic criteria evidence [44]. This has led to its received skepticism by modern medicine society due to the lack of high quality trials. In previous reviews, there were basically no studies related to PPI de-escalation was retrieved and recorded, therefore in this trial, we will conduct a rigorous randomized, double-blind clinical trials to clarify the efficacy of TCM granules derived from TJ methodology combined with PPIs de-escalation, and will perform quality of life, mental health, recurrence and PPI withdrawal rate of the therapy.

We regard this trial with upmost importance due to the facts that $20-30 \%$ of NERD patients do not respond to PPIs treatment [45] and that the long-term use of PPIs produces dependence and negative side effects [46, 47]. With the cooperation of TCM granules and PPIs de-escalation therapy, it can synergistically adjust the holistic internal and external energy of NERD patients. On the premise of relieving symptoms, gradually reduce the dosage of acid suppressive drugs every two weeks to withdraw. It can provide a solution to the problem of NERD patients not responding to PPIs and reduce the side effects caused by long-term use of PPIs. Experiments had proved of the effect of granules based on TJ methodology on increasing the level of plasma motilin, reducing the level of gastric acid, and promoting the gastric emptying. The acute toxicity experiment showed that there was no toxic reaction in mice [23].

This trial will use VAS for heartburn and regurgitation, major and minor symptoms scale, SF-36, PRO, SAS, SDS, GERD-HRQL, TCM syndromes scales, symptom recurrence rate to measure physiological and psychological outcomes to comprehensively evaluate the changes imposed by this therapy for the participants before, during and after treatment and the PPIs withdrawal rate. In order to provide high-level medical evidence for the TCM treatment of NERD and to form a new therapy regimen for NERD. For patients with long-term use of PPIs that are unable to withdraw, the advantages of the new therapy and the mechanisms related to gut microbiota will be evaluated. This trial is expected to solve the side effects and dependence caused by long-term use of PPIs, and compare the gut microbiota diversity of healthy volunteers and NERD patients before and after treatment to prepare for the next step of exploring the underlying mechanism of the disease. The results of this trial will help to improve the quality of evidence of TCM studies and the clinical efficacy of NERD treatments, reduce dependence on PPIs, and improve the quality of life of patients, ideally yielding good social benefits.

\section{Trial Status}


protocol version number and date: XYYY-KY-NERD-03, 22th Feb, 2020

the date recruitment began: 15th Jun, 2020

the approximate date when recruitment will be completed: 31nd Dec, 2022

\section{Abbreviations}

$\mathrm{AET}=$ acid exposure time, $\mathrm{BE}=$ Barrett's oesophagus, $\mathrm{BP}=$ Bodily Pain, $\mathrm{EE}=$ erosive esophagitis, GERD $=$ Gastroesophageal reflux disease, GERD-HRQL=the Gastroesophageal Reflux Disease-Health-Related Quality-Of-Life, GH=General Health, HT=Health Transition, MH=Mental Health, NERD=Non-erosive reflux disease, $\mathrm{PF}=$ Physical Functioning, PPIs=Proton pump inhibitors, PRO=Patient-Reported Outcome, RCT=randomized controlled trial, RE=Role-Emotiona, RP=Role-Physical, SAS=Self-rating Anxiety Scale, SDS=Self-rating Depression Scale, SF=Social Functioning, SF-36=the MOS 36-item Short From Health Survey, TCM=Traditional Chinese medicine, TJ=Tongjiang, VAS=Visual Analogue Scale, VT=Vitality

\section{Declarations}

\section{Ethics approval and consent to participate}

This trial has been approved by The Ethical Review Committee of Xiyuan Hospital of China Academy of Chinese (Approval Number: 2020XLA008-2). Before any data collection, randomization and data analysis, written informed consent must be obtained from all participants.

\section{Consent for publication:}

Not applicable.

\section{Availability of data and materials[}

Not applicable. This is a protocol, no data generated.

\section{Competing interests:}

None.

\section{Funding]}

National Key Research and Development Project (No. 2019YFC1709600)

\section{Authors' contributions:}

Conceptualization and framework of the study: Xudong Tang, Haomeng Wu, Xia Li, Ting Chen; Preparation for funding application: Haomeng Wu, Xia Li, Jiaqi Zhang, Ting Chen, Beihua Zhang; Preparing ethical materials and applications: Jinxin Ma, Beihua Zhang; Preparing the manuscript: Xia Li, Haomeng Wu, Ting Chen, Xiaoshuang Shi; Reviewing the manuscript: Xudong Tang, Fengyun Wang. All authors read and approved the final manuscript. 
The authors would like to acknowledge the staff who participated in the project design and implementation.

\section{References}

1. Kethman W, Hawn M. New Approaches to Gastroesophageal Reflux Disease. J Gastrointest Surg. 2017;21(9):1544-52. doi:10.1007/s11605-017-3439-5.

2. Savarino E, de Bortoli N, De Cassan C, Della Coletta M, Bartolo O, Furnari M, et al. The natural history of gastro-esophageal reflux disease: a comprehensive review. Dis Esophagus. 2017;30(2):1-9. doi:10.1111/dote.12511.

3. Nikaki K, Woodland P, Sifrim D. Adult and paediatric GERD: diagnosis, phenotypes and avoidance of excess treatments. Nat Rev Gastroenterol Hepatol. 2016;13(9):529-42. doi:10.1038/nrgastro.2016.109.

4. Gyawali CP, Kahrilas PJ, Savarino E, Zerbib F, Mion F, Smout A, et al. Modern diagnosis of GERD: the Lyon Consensus. Gut. 2018;67(7):1351-62. doi:10.1136/gutjnl-2017-314722.

5. Aziz Q, Fass R, Gyawali CP, Miwa H, Pandolfino JE, Zerbib F. Functional Esophageal Disorders Gastroenterology. 2016. doi:10.1053/j.gastro.2016.02.012.

6. Savarino E, Tutuian R, Zentilin P, Dulbecco P, Pohl D, Marabotto E, et al. Characteristics of reflux episodes and symptom association in patients with erosive esophagitis and nonerosive reflux disease: study using combined impedance-pH off therapy. Am J Gastroenterol. 2010;105(5):1053-61. doi:10.1038/ajg.2009.670.

7. Papa A, Urgesi R, Grillo A, Danese S, Guglielmo S, Roberto I, et al. Pathophysiology, diagnosis and treatment of non-erosive reflux disease (NERD). Minerva Gastroenterol Dietol. 2004;50(3):215-26.

8. de Bortoli N, Ottonello A, Zerbib F, Sifrim D, Gyawali CP, Savarino E. Between GERD and NERD: the relevance of weakly acidic reflux. Ann N Y Acad Sci. 2016;1380(1):218-29. doi:10.1111/nyas.13169.

9. Rinsma NF, Farré R, Troost FJ, Elizalde M, Keszthelyi D, Helyes Z, et al. Exploration of the Esophageal Mucosal Barrier in Non-Erosive Reflux Disease. Int J Mol Sci. 2017;18(5):1091. doi:10.3390/ijms18051091.

10. Kawami N, Iwakiri K, Sakamoto C. [Pathophysiology and Treatment of PPI-resistant NERD]. Nihon Rinsho. 2015;73(7):1197-201.

11. Katz PO, Zavala S. Proton pump inhibitors in the management of GERD. J Gastrointest Surg. 2010;14(Suppl 1):62-6. doi:10.1007/s11605-009-1015-3.

12. Fass R, Sifrim D. Management of heartburn not responding to proton pump inhibitors. Gut. 2009;58(2):295309. doi:10.1136/gut.2007.145581.

13. Kinoshita Y, Ashida K, Hongo M. Randomised clinical trial: a multicentre, double-blind, placebo-controlled study on the efficacy and safety of rabeprazole $5 \mathrm{mg}$ or $10 \mathrm{mg}$ once daily in patients with non-erosive reflux disease. Aliment Pharmacol Ther. 2011;33(2):213-24. doi:10.1111/j.1365-2036.2010.04508.x.

14. Heidelbaugh JJ, Kim AH, Chang R, Walker PC. Overutilization of proton-pump inhibitors: what the clinician needs to know. Therap Adv Gastroenterol. 2012;5(4):219-32. doi:10.1177/1756283x12437358.

15. Malfertheiner P, Kandulski A, Venerito M. Proton-pump inhibitors: understanding the complications and risks. Nature Reviews Gastroenterology Hepatology. 2017;14(12):697-710. doi:10.1038/nrgastro.2017.117.

16. Bavishi C, Dupont HL. Systematic review: the use of proton pump inhibitors and increased susceptibility to enteric infection. Aliment Pharmacol Ther. 2011;34(11-12):1269-81. doi:10.1111/j.1365- 
2036.2011.04874.x.

17. Bloom BS, Jayadevappa R, Wahl P, Cacciamanni J. Time trends in cost of caring for people with gastroesophageal reflux disease. Am J Gastroenterol. 2001;96(8 Suppl):64-9. doi:10.1016/s00029270(01)02587-4.

18. Reimer C, Bytzer P. Discontinuation of long-term proton pump inhibitor therapy in primary care patients: a randomized placebo-controlled trial in patients with symptom relapse. Eur J Gastroenterol Hepatol. 2010;22(10):1182-8. doi:10.1097/MEG.0b013e32833d56d1.

19. Salehi M, Karegar-Borzi H, Karimi M, Rahimi R. Medicinal Plants for Management of Gastroesophageal Reflux Disease: A Review of Animal and Human Studies. J Altern Complement Med. 2017;23(2):82-95. doi:10.1089/acm.2016.0233.

20. Yoon SL, Grundmann O, Smith KF, Mason SR. Dietary Supplement and Complementary and Alternative Medicine Use Are Highly Prevalent in Patients with Gastrointestinal Disorders: Results from an Online Survey. J Diet Suppl. 2019;16(6):635-48. doi:10.1080/19390211.2018.1472712.

21. Xiao J, Yang Y, Zhu Y, Qin Y, Li Y, Fu M, et al. Efficacy and Safety of Traditional Chinese Medicine on Nonerosive Reflux Disease: A Meta-Analysis of Randomized Controlled Trials. Evid Based Complement Alternat Med. 2018;2018:1505394. doi:10.1155/2018/1505394.

22. Teschke R, Wolff A, Frenzel C, Eickhoff A, Schulze J. Herbal traditional Chinese medicine and its evidence base in gastrointestinal disorders. World J Gastroenterol. 2015;21(15):4466-90. doi:10.3748/wjg.v21.i15.4466.

23. Li BS, Li ZH, Tang XD, Zhang LY, Zhao YP, Bian LQ, et al. A randomized, controlled, double-blinded and double-dummy trial of the effect of tongjiang granule on the nonerosive reflux disease of and Gan-Wei incoordination syndrome. Chin J Integr Med. 2011;17(5):339-45. doi:10.1007/s11655-011-0724-0.

24. Chan AW, Tetzlaff JM, Altman DG, Laupacis A, Gøtzsche PC, Krleža-Jerić K, et al. SPIRIT 2013 statement: defining standard protocol items for clinical trials. Ann Intern Med. 2013;158(3):200-7. doi:10.7326/00034819-158-3-201302050-00583.

25. Xu TT, Zhu SL. The clinical curative effect by Shugan Hewei Decoction on the treatment of NERD. Lishizhen Medicine Materia Medica Research. 2017;28(05):1135-6. [Chinese].

26. Tong C, Xu K. Effects of rabeprazole enteric-coated tablets on the number of reflux and serum LPO in patients with non-erosive gastroesophageal reflux disease. Journal of Nantong University (Medical Sciences). 2016;36(06):620-2. [Chinese].

27. Aitken RC. Measurement of feelings using visual analogue scales. Proc R Soc Med. 1969;62(10):989-93.

28. Gerich J. Visual analogue scales for mode-independent measurement in self-administered questionnaires. Behav Res Methods. 2007;39(4):985-92. doi:10.3758/bf03192994.

29. Lee JS, Suh KT, Kim JI, Lee HS, Goh TS. Validation of the korean version of the neck pain and disability scale. Asian spine journal. 2013;7(3):178-83. doi:10.4184/asj.2013.7.3.178.

30. Ware JE. SF-36 Health Survey: Manual and Interpretation Guide. Health Institute; 1993.

31. Perneger TV, Leplège A, Etter JF, Rougemont A. Validation of a French-language version of the MOS 36-Item Short Form Health Survey (SF-36) in young healthy adults. J Clin Epidemiol. 1995;48(8):1051-60. doi:10.1016/0895-4356(94)00227-h. 
32. Guillemin F, Bombardier C, Beaton D. Cross-cultural adaptation of health-related quality of life measures: literature review and proposed guidelines. J Clin Epidemiol. 1993;46(12):1417-32. doi:10.1016/08954356(93)90142-n.

33. Gandek B, Ware JE Jr. Methods for validating and norming translations of health status questionnaires: the IQOLA Project approach. International Quality of Life Assessment. J Clin Epidemiol. 1998;51(11):953-9. doi:10.1016/s0895-4356(98)00086-9.

34. Tang XD, Wang P, Zhao YP, Bian LQ, et al. Experts Questionnaires Survey on the Applicability for FGIDs of Patient Reported Outcomes Instrument for Chronic Gastrointestinal Disease. Chin J Integr Tradit West Med (Chin). 2018;38(02):182-5. [Chinese].

35. Tang XD, Wang FY, Bian LQ. Development of FGIDs gastrointestinal symptom overlap and patient report outcome scale. J Tradit Chin Med. 2015;56(21):1826-30. [Chinese].

36. Li H, Jin D, Qiao F, Chen J, Gong J. Relationship between the Self-Rating Anxiety Scale score and the success rate of 64-slice computed tomography coronary angiography. Int J Psychiatry Med. 2016;51(1):4755. doi:10.1177/0091217415621265.

37. Zung WW, Magruder-Habib K, Velez R, Alling W. The comorbidity of anxiety and depression in general medical patients: a longitudinal study. J Clin Psychiatry. 1990;51 Suppl:77-80. discussion 1.

38. Dunstan DA, Scott N. Clarification of the cut-off score for Zung's self-rating depression scale. BMC Psychiatry. 2019;19(1):177. doi:10.1186/s12888-019-2161-0.

39. Wang CF, Cai ZH, Xu Q. Evaluation analysis of self-rating depression disorder scale in 1,340 people. Chin J Nervous Mental Dis. 2009;12:267-8.

40. Velanovich V. The development of the GERD-HRQL symptom severity instrument. Dis Esophagus. 2007;20(2):130-4. doi:10.1111/j.1442-2050.2007.00658.x.

41. Pilone V, Tramontano S, Renzulli M, Zulli C, Schiavo L. Gastroesophageal Reflux After Sleeve Gastrectomy: New Onset and Effect on Symptoms on a Prospective Evaluation.

42. Shi X, Li X, Ma J, Che H, Ma X, Xie J, et al. Chinese Medicine JQ Granule Combined with Half-dose Omeprazole for Nonerosive Reflux Disease: A Multicenter, Randomized, Double-blind, Placebo-controlled Trial Study Protocol. European Journal of Integrative Medicine. 2019;31:100974. doi:10.1016/j.eujim.2019.100974.

43. Sandhu DS, Fass R. Current Trends in the Management of Gastroesophageal Reflux Disease. Gut Liver. 2018;12(1):7-16. doi:10.5009/gnl16615.

44. Ling W, Li Y, Jiang W, Sui Y, Zhao HL. Common Mechanism of Pathogenesis in Gastrointestinal Diseases Implied by Consistent Efficacy of Single Chinese Medicine Formula: A PRISMA-Compliant Systematic Review and Meta-Analysis. Medicine. 2015;94(27):e1111. doi:10.1097/md.0000000000001111.

45. Yadlapati R, DeLay K. Proton Pump Inhibitor-Refractory Gastroesophageal Reflux Disease. Med Clin North Am. 2019;103(1):15-27. doi:10.1016/j.mcna.2018.08.002.

46. Casciaro M, Navarra M, Inferrera G, Liotta M, Gangemi S, Minciullo PL. PPI adverse drugs reactions: a retrospective study. Clin Mol Allergy. 2019;17(1):1. doi:10.1186/s12948-019-0104-4.

47. Gyawali CP, Fass R. Management of Gastroesophageal Reflux Disease. Gastroenterology. 2018;154(2):30218. doi:10.1053/j.gastro.2017.07.049. 


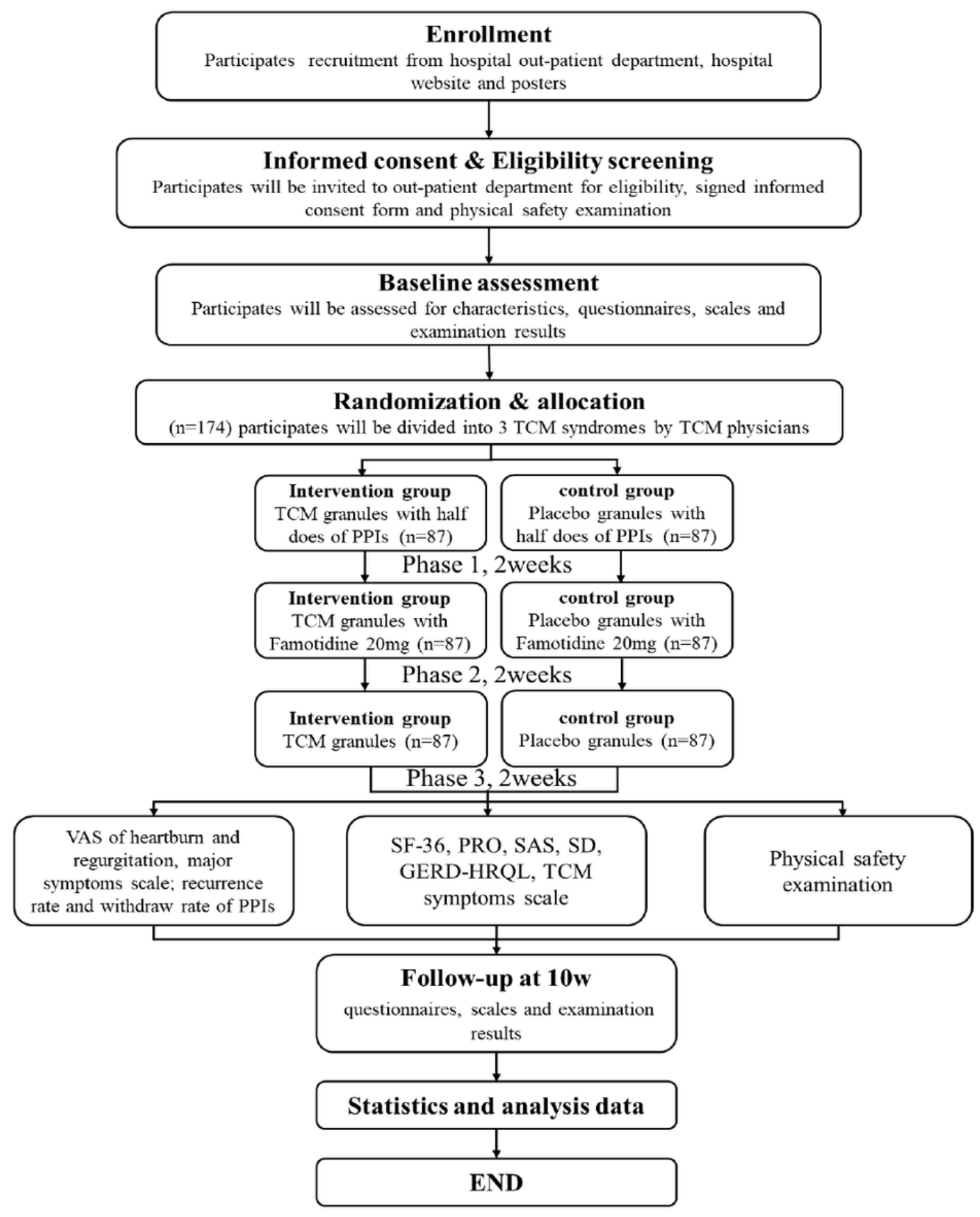

Figure 1

Flowchart of the proposed trial. VAS, visual analogue scale; TCM, traditional Chinese medicine; SF-36, the MOS 36-item Short From Health Survey; PRO, Patient-Reported Outcome; SAS, Self-rating Anxiety Scale; SDS, Selfrating Depression Scale; GERD-HRQL, the Gastroesophageal Reflux Disease-Health-Related Quality-Of-Life.

\section{Supplementary Files}


This is a list of supplementary files associated with this preprint. Click to download.

- SPIRITChecklist.doc 